\title{
Avian genome evolution: insights from a linkage map of the blue tit (Cyanistes caeruleus)
}

\author{
B Hansson ${ }^{1}$, M Ljungqvist ${ }^{1}$, DA Dawson ${ }^{2}$, JC Mueller ${ }^{3}$, J Olano-Marin ${ }^{3}$, H Ellegren $^{4}$ and J-Å Nilsson ${ }^{1}$ \\ ${ }^{1}$ Department of Animal Ecology, Lund University, Lund, Sweden; ${ }^{2}$ Department of Animal and Plant Sciences, University of Sheffield, \\ Sheffield, UK; ${ }^{3}$ Department of Behavioural Ecology E Evolutionary Genetics, Max Planck Institute for Ornithology, Starnberg \\ (Seewiesen), Germany; ${ }^{4}$ Department of Evolutionary Biology, Evolutionary Biology Centre, Uppsala University, Uppsala, Sweden
}

\begin{abstract}
We provide a first-generation linkage map of the blue tit (Cyanistes caeruleus), a passerine within the previously genetically uncharacterized family Paridae, which includes 91 orthologous loci with a single anchored position in the chicken (Gallus gallus) sequence assembly. The map consists of 18 linkage groups and covers $935 \mathrm{cM}$. There was highly conserved synteny between blue tit and chicken with the exception of a split on chromosome 1, potential splits on chromosome 4 and the translocation of two markers from chromosome 2 and 3, respectively, to chromosome 5 . Gene order was very well conserved for the majority of chromosomes, an exception being chromosome 1 where multiple rearrangements were detected. Similar results were
\end{abstract}

obtained in a comparison to the zebra finch (Taeniopygia guttata) genome assembly. The recombination rate in females was slightly higher than in males, implying a moderate degree of heterochiasmy in the blue tit. The map distance of the blue tit was $\sim 78 \%$ of that of the Wageningen chicken broiler population, and very similar to the Uppsala chicken mapping population, over homologous genome regions. Apart from providing insights into avian recombination and genome evolution, our blue tit linkage map forms a valuable genetic resource for ecological and evolutionary research in Paridae.

Heredity (2010) 104, 67-78; doi:10.1038/hdy.2009.107; published online 26 August 2009

Keywords: intronic single nucleotide polymorphisms; linkage; microsatellite; passerine; recombination; synteny

\section{Introduction}

The construction of genetic maps or linkage maps dates back to Sturtevant's pioneering study on recombination between sex-linked genes in Drosophila that laid out the foundation and logic for linkage mapping (Sturtevant, 1913). Since then, detailed linkage maps have been attained for a large number of model organisms and domestic animals and plants. A main aim in ecological and evolutionary genetic research is to disentangle the genetics of phenotypic variation and fitness in the wild to achieve a detailed understanding of processes, such as the mode and speed of phenotypic responses to selection and the importance of interactions between genes (for example, Steiner et al. (2007); Gratten et al. (2008); reviewed in Ellegren and Sheldon (2008)). However, genomic resources, such as the recombination-based linkage maps necessary for this kind of research, are still lacking for a majority of ecologically and evolutionary model organisms, including most wild bird species.

The genome structure of birds is characterized by unusually high chromosome number and a complex karyotype, consisting of several macrochromosomes and numerous microchromosomes. Cytogenetic analyses using fluorescent in situ hybridization of chromosome-

Correspondence: Dr B Hansson, Department of Animal Ecology, Lund University, Ecology Building, Lund University, Lund, S-223 62, Sweden. E-mail: bengt.hansson@zooekol.lu.se

Received 8 January 2009; revised 24 June 2009; accepted 29 June 2009; published online 26 August 2009 specific probes have shown that this complex genome structure is a shared feature of many avian lineages, including Galliformes and Passeriformes. Despite 80-100 million years of independent evolution (Shetty et al., 1999; van Tuinen et al., 2000), the Galliformes and Passeriformes have a highly conserved genome structure with few interchromosomal rearrangements, at least as far as can be revealed by cytogenetic resolution (Shields, 1982; Derjusheva et al., 2004; Itoh and Arnold, 2005; Griffin et al., 2007). The release of the genome sequence assembly of the red jungle fowl (Gallus gallus) in 2004 (International Chicken Genome Sequencing Consortium, 2004) provided a unique possibility to study avian genome evolution in more detail and has triggered avian molecular-based research (Ellegren, 2005). Comparative gene mapping between chicken and the great reed warbler (Acrocephalus arundinaceus), using the first constructed linkage map in passerine birds, confirmed that the gene content on the chromosomes, that is, synteny, has been highly conserved between the Galliformes and Passeriformes, and further revealed a high degree of gene-order conservation within chromosomes (Hansson et al., 2005; Dawson et al., 2006, 2007; Ảkesson et al., 2007). Recently, these conclusions were supported and further substantiated by studies of more extensive genetic maps of two other passerines, the zebra finch (Taeniopygia guttata) (Stapley et al., 2008) and the collared flycatcher (Ficedula albicollis) (Backström et al., 2006, 2008b). The higher marker density and genome coverage of these linkage maps provided increased resolution for comparative gene mapping, and a few novel interchromosomal 
translocations were detected, as were several previously undetected intrachromosomal rearrangements (Backström et al., 2006, 2008b; Stapley et al., 2008).

In addition to the obvious relevance of linkage maps in comparative studies of genome evolution, they also provide opportunities to study the rate and pattern of recombination between species. In comparison to mammals, chicken has a higher recombination rate per base pair, especially over the microchromosomes and in purebred domestic populations (International Chicken Genome Sequencing Consortium, 2004; Groenen et al., 2009). Moreover, the rate of recombination is similar in cocks and hens (Groenen et al., 1998, 2009), whereas sexbiased recombination, that is, heterochiasmy, is a wellknown phenomenon among mammals, including human and mouse (Dietrich et al., 1996; Kong et al., 2002; reviewed in Lenormand and Dutheil, 2005). The passerine linkage maps suggest recombination rates that are lower than that in the domestic chicken and more similar to mammals, with interesting variation between species. In the great reed warbler, the linkage map intervals are less than a fifth of those of the chicken (Dawson et al., 2007), and for zebra finches and collared flycatchers approximately a fourth and a half, respectively (Stapley et al., 2008; Backström et al., 2008b). However, it is noted that these ratios need to be adjusted as high-resolution mapping in chicken recently resulted in reduced map distances, especially for crosses between more divergent breeds (Groenen et al., 2009). The degree of heterochiasmy varies considerably between passerines from a strong female-biased recombination rate in the great reed warbler (Hansson et al., 2005; Åkesson et al., 2007) to a moderate male-biased rate in the collared flycatcher (Backström et al., 2008b). Evaluating to what degree these different recombination characteristics are shared among passerines would be valuable, as it may yield important insights in the evolution of heterochiasmy (Lenormand and Dutheil, 2005).

In this study, we provide a first-generation linkage map of the blue tit (Cyanistes caeruleus), a passerine within the previously genetically uncharacterized passerine family Paridae. The linkage map was constructed by genotyping a pedigree in a Swedish population at a set of 98 polymorphic microsatellites and intronic single nucleotide polymorphisms (SNPs), of which 91 and 94 have an orthologue with a single anchored position in the chicken (International Chicken Genome Sequencing Consortium, 2004; http://www.ncbi.nlm.nih.gov/ mapview/) and zebra finch (http://genome.wustl.edu/ genomes/view/taeniopygia_guttata/; http://www.ncbi. nlm.nih.gov/mapview/) genome assemblies, respectively (cf. Dawson et al., 2007; Backström et al., 2008a; Olano-Marin et al., 2009). This genotyping strategy enables comparisons of synteny, gene order and pattern of recombination in homologous chromosomal regions of Passeriformes and Galliformes, two phylogenetically highly diverged avian lineages (cf. Dawson et al., 2007; Backström et al., 2008b).

The blue tit is an important avian ecological model species with research in several Western Palaearctic populations focusing on topics including physiology (Nager and Wiersma, 1996), phylogeography (Kvist et al., 2005), inbreeding (Foerster et al., 2003), parasite-host coevolution (Stjernman et al., 2004), quantitative genetics
(Råberg et al., 2003), microevolution (Charmantier et al., 2004), natural selection (Blondel et al., 1999) and sexual selection (Petrie and Kempenaers, 1998). A genetic map of the blue tit is useful to the wider research community, as it opens up possibilities of conducting research on evolutionary aspects of the species that so far have not been addressed, such as, for example, the degree of linkage disequilibrium in different populations and the genetic basis of quantitative traits. Furthermore, recombination and mapping data of a species of Paridae are important for understanding the usefulness of the chicken and zebra finch genome assemblies for passerine evolutionary genetical research in general (cf. Slate, 2005; Ellegren and Sheldon, 2008).

\section{Materials and methods}

\section{Blue tit genotyping}

The blue tit is a small-sized passerine of the family Paridae. The species breeds across much of the Western Palaearctic and is either a resident or performs a shortdistance migration (Cramp, 1992). Its karyotype is not yet described, but other studied passerines have similar numbers of macro- and microchromosomes $(2 n=78-80$; Derjusheva et al., 2004; Itoh and Arnold, 2005) as chicken $(2 n=78$; Masabanda et al., 2004). As in all other bird species, male blue tits are homogametic (ZZ), whereas females are heterogametic (ZW).

In this study, we conducted linkage mapping in a nestbox population of blue tits in the Revinge area, southern Sweden $\left(55^{\circ} 41^{\prime} \mathrm{N}, 13^{\circ} 26^{\prime} \mathrm{E}\right)$, where a detailed ecological study has been ongoing since 1983 (for example, Nilsson and Svensson (1993); Råberg et al. (2003); Stjernman et al. (2004)). We collected blood samples and extracted DNA (using an ammonium acetate protocol) from a set of large families containing between 10 and 17 individuals breeding in 2005 and 2007. In total, the pedigree included 525 individuals from 34 families spanning over two to three generations. Parentage and sex were genetically determined, and cases of extra-pair parentage were detected (by allelic mismatches on several markers) and accounted for in all broods.

We initially tested for microsatellite polymorphism among four unrelated blue tits in the study population by screening a large number of described microsatellite loci (cf. Hansson et al., 2005; Dawson et al., 2007; OlanoMarin et al., 2009). In addition, intronic SNPs were detected with the comparative anchor-tagged sequences approach (Lyons et al., 1997), in which introns of two unrelated individuals were sequenced using a set of gene-based exon-primed intron-crossing primers developed by Backström et al. (2008a). In total, 103 SNPs in 53 genes were detected (Backström et al., 2008a, Hansson B and Ellegren $\mathrm{H}$, unpublished data). In the mapping families, we genotyped 98 polymorphic markers of which 91 (61 microsatellites and 30 intronic SNPs) and 94 (64 microsatellites and 30 intronic SNPs) had a singlecopy orthologue in the chicken and zebra finch genome sequence assembly, respectively (as determined by BLAST searches; see below) (Supplementary Table 1). Four markers were anonymous microsatellite loci for which we could not find single-copy orthologues in chicken or zebra finch (Supplementary Table 1). Primer sequences and PCR conditions of the microsatellites are 
given in Supplementary Table 1. The PCR products of the microsatellites were separated and visualized using an ABI 3730 or an ABI 3130 capillary sequencer (Applied Biosystems, Inc., Foster City, CA, USA (cf. Dawson et al., 2007). Microsatellite data were analysed in GENEMAPPER 3.0 (Applied Biosystems). The intronic SNPs were genotyped using MALDI-TOF Mass spectrometry (Sequenom Mass Array; Sequenom, Inc., San Diego, CA, USA). Null alleles were present at a few loci in some families (estimated null-allele frequencies at the population level are given in Olano-Marin et al. (2009)), and the segregation of these alleles was accounted for in the analyses.

\section{Linkage map building}

Linkage groups were constructed in CRIMAP 2.4 (Lander and Green, 1987). This program calculates two-point recombination fractions, provides logarithmic odds ratio (LOD) scores for recombination estimates, and tests marker order. We assigned autosomal markers to linkage groups by calculating the recombination fractions between all pairs of markers with the TWOPOINT option in CRIMAP; markers were considered as significantly linked at a threshold of LOD $>3.0$ (that is, markers are $>1000$ times more likely to be linked at the estimated recombination rate than to be unlinked). A few markers (10005, 25442, CcaTgu16, PmaC25, TG13-009, TG13-017 and $\mathrm{VeCr} 02$; many having few informative meioses, Supplementary Table 1) could not be assigned to any other marker at this threshold, but were linked to one or more markers in a single linkage group at a less stringent threshold of LOD $>2.0$ (that is, linkage $>100$ times more likely than no linkage). As none of these markers were linked to more than one linkage group, and thus no conflicting assignments occurred, we included all of them in the linkage map analyses. The Z-linked loci (Ase46, CcaTgu31, Phtr3, Tgu9, TGZ-037 and TGZ-040) were assigned to this chromosome on the basis of every female (being hemizygous, ZW) having only a single allele, whereas males were heterozygous or homozygous. We determined the most parsimonious ordering of markers within each linkage group (the order with the highest likelihood support) and the framework order (that is, the order of markers with a single significant map position at a LOD score of 3.0), with the options BUILD, FLIPSN and FIXED. Heterochiasmy was evaluated by running sex-specific analyses. Map distances are given in Kosambi centiMorgans (cM).

\section{Detection of homologous single-copy chicken-passerine sequences}

All SNPs were located in introns of genes with a single position in the chicken genome (Backström et al., 2008a). We confirmed that the amplified intronic blue tit sequences corresponded to the expected chicken gene by conducting BLAST searches against the chicken genome assembly ('WASHU 2.1'; http://pre.ensembl. org/Gallus_gallus/index.html). The position was confirmed at an $E$-value of $<1 \times 10^{-10}$ for all loci, with the exception of locus 09974 that had several significant hits (data not shown). The position of the homologous genes in the zebra finch genome (Build 1.1; taeGut3.2.4) came from Ensembl.org (for example, for ENSGALG
00000016379: http://www.ensembl.org/Gallus_gallus/ Gene/Compara_Alignments?g=ENSGALG00000016379).

To detect the position of the microsatellite loci, we conducted BLAST analyses following the procedures of Dawson et al. (2007). In short, we performed a BLAST search of each microsatellite sequence against the chicken genome with the Ensembl WU-Blast software (Gish W. 1996-2004; http://blast.wustl.edu) using the Ensembl chicken genome browser and the 'distant homologies' search setting, which is optimal for detecting homology between divergent taxa (http://www. ensembl.org/Gallus_gallus/blastview). We used a word size of 9, and accepted matches with an E-value of $<1 \times 10^{-10}$; when more than one significant match occurred, the best hit had to be $<1 \times 10^{-10}$ and the next hit had to be at least $1 \times 10^{-10}$ weaker.

Furthermore, five microsatellite loci that could not be directly located in the chicken genome were mapped using zebra finch sequence data following Dawson et al. (2007). Homologous zebra finch sequences were identified by performing a cross-species megaBLAST search of the loci against the NCBI's zebra finch WGS trace archive database (http:/ / www.ncbi.nlm.nih.gov/projects/genome/ seq/BlastGen/BlastGen.cgi?pid =12898). The homologous zebra finch sequences were much longer (ca. 700-900 bp) than the original microsatellite sequences and, therefore, when matched against the chicken genome ('WASHU 2.1'; as above), several of them could be assigned a location in the chicken genome (Supplementary Table 1).

To assign locations for the microsatellite markers in the zebra finch genome we performed a BLAT search of each microsatellite sequence against the zebra finch genome (Build 1.1; taeGut3.2.4) at the UCSC browser (http:// genome.ucsc.edu/cgi-bin/hgGateway) or by using a BLASTN search of each microsatellite against the zebra finch genome in the GSC BLAST server at the Washington University in St Louis (WUSTL) School of Medicine (http://genome.wustl.edu/tools/blast/) (details given in Olano-Marin et al., 2009).

\section{Linkage map distances in chicken}

A comparison of the recombination rate between blue tit and chicken requires data on linkage map distances in chicken for the chromosomal regions mapped in blue tit. For this comparison, we focused on chromosomes with a high degree of synteny and gene-order conservation, and substantial coverage ( $>50 \%$ of the chicken chromosome). Therefore, these analyses included data from the homologous parts of eight chromosomes $(G g a 2,5,6,7,8,11,20$ and Z). As the orthologues of the passerine microsatellite loci are not included on the chicken genetic linkage map, we used data for the closest chicken marker with a linkage map location in the chicken genome (WUR linkage map; http://www.ncbi. nlm.nih.gov/genome/guide/chicken/) and corrected the distances according to recent high-resolution mapping data of the Wageningen broiler chicken population (total map size of ca. $3325 \mathrm{cM}$ ) and the Uppsala chicken mapping population (total map size of ca. $2790 \mathrm{cM}$; Groenen et al., 2009). In 15 of 16 cases the marker used for determining the linkage map interval in chicken was within $2 \mathrm{Mbp}$ from the orthologous locus mapped in blue tit; the exception being the chicken marker, LEI0346 (at the end of chromosome 20), which 
was located $5.5 \mathrm{Mbp}$ from the position of the orthologous locus (PmaGAn30).

\section{Results}

Linkage map and heterochiasmy

The two-point analyses detected that 89 of the 98 markers were linked to at least one other marker in our blue tit mapping data set. These markers built up 18 linkage groups with 2-17 markers (Figure 1; Supplementary Table 1). No markers were linked to more than one linkage group, and thus no conflicting assignments occurred. The parsimonious sex-average autosomal linkage map spanned $935 \mathrm{~cm}$. There was a moderate degree of heterochiasmy, with a female map of $1046 \mathrm{cM}$ and a male map of $887 \mathrm{cM}$, that is, a female-to-male map ratio of 1.18 (Wilcoxon sign rank test: $z=1.50, n=18$

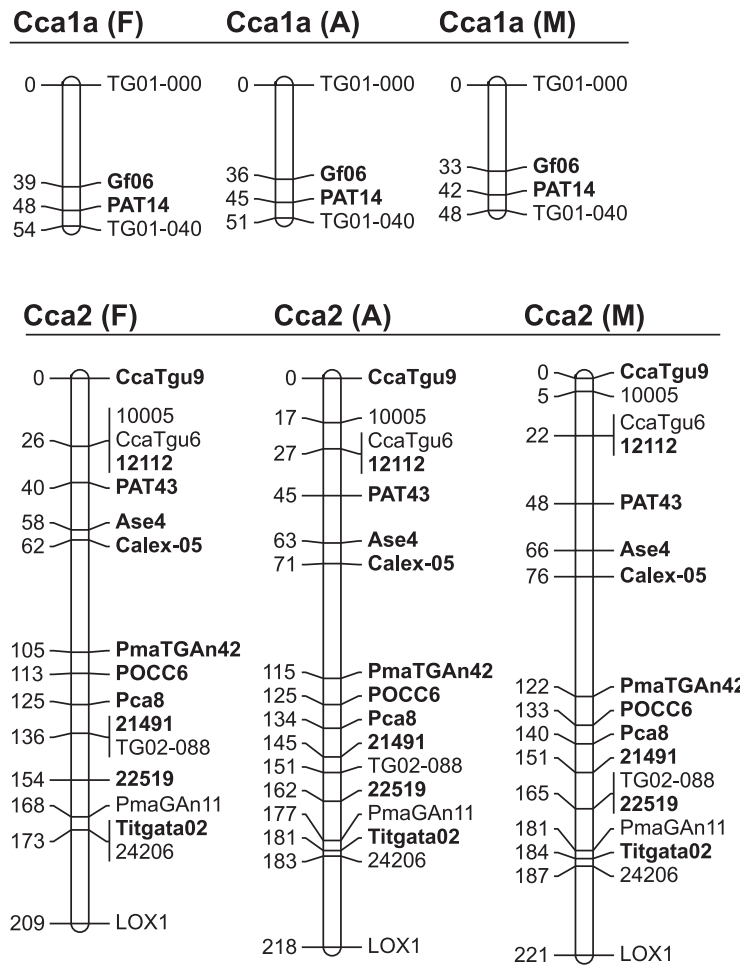

Cca4a (F) Cca4a (A) Cca4a (M)

$0-\left.\bigcirc\right|_{\mathrm{TG04-061}} ^{16214} 0-\left.0\right|_{\mathrm{TG} 04-061} ^{16214} 0-\left.O\right|_{\mathrm{TG} 04-061} ^{16214}$

Cca4b (F) Cca4b (A) Cca4b (M)

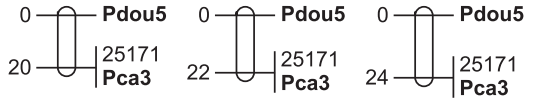

$\frac{\text { Cca4c }}{0-0-\text { MJG1 }} \frac{\text { Cca4d }}{0-0-12303} \frac{\text { Cca4e }}{0-O-25924}$

Cca1b (F) Cca1b (A) Cca1b (M)
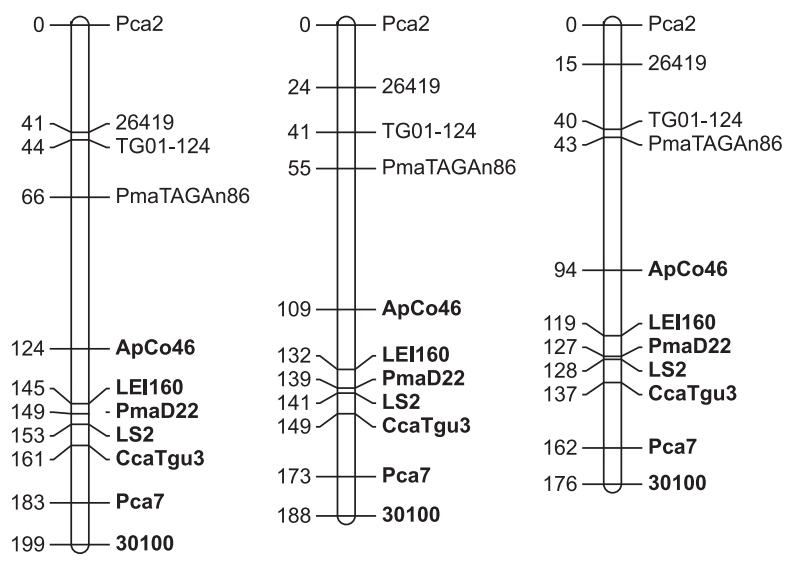

Cca3a (F) Cca3a (A) Cca3a (M)

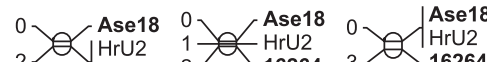

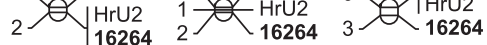

Cca3b (F) Cca3b (A) Cca3b (M)

Cca3c

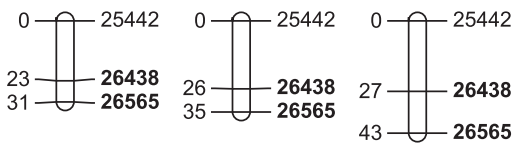

$0 \multimap$ Ase 60

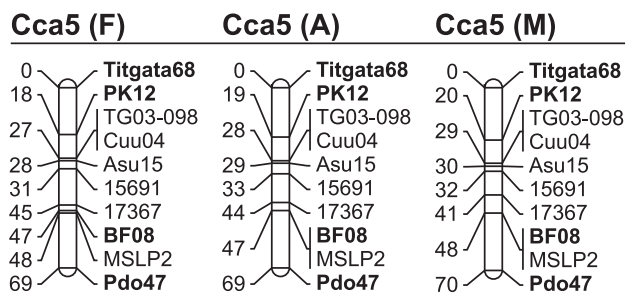

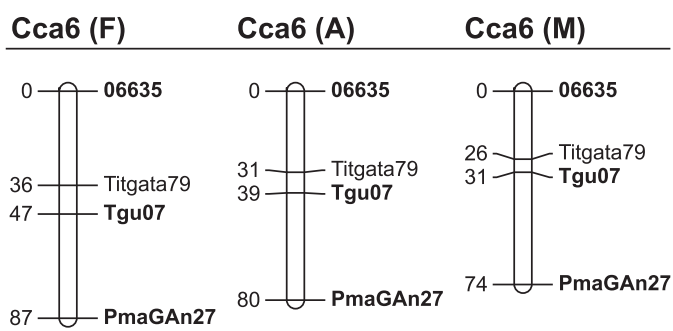

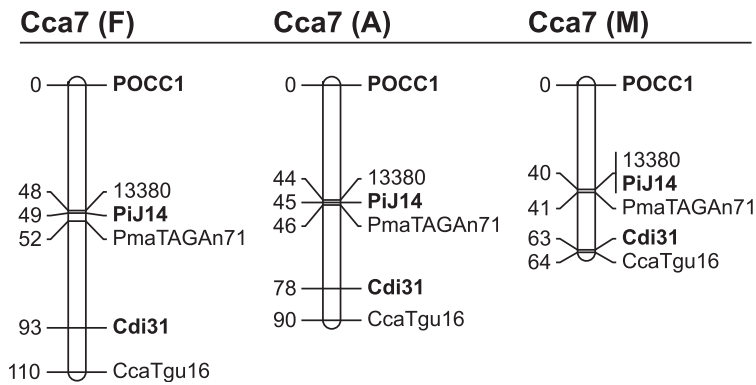

Figure 1 Sex-specific and sex-average linkage maps of the blue tit (Cyanistes caeruleus). Female linkage groups are to the left (for example, Ccala $(\mathrm{F})$ ) and male maps to the right (for example, Cca1a (M)) in each linkage group. Shown is the most parsimonious map, with the framework loci (that is, loci with an unambiguous position on the map) in bold font. Genetic distances are given in cM. 


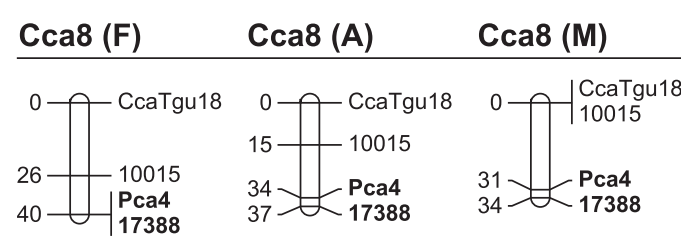

Cca11 (F) Cca11 (A) Cca11 (M)
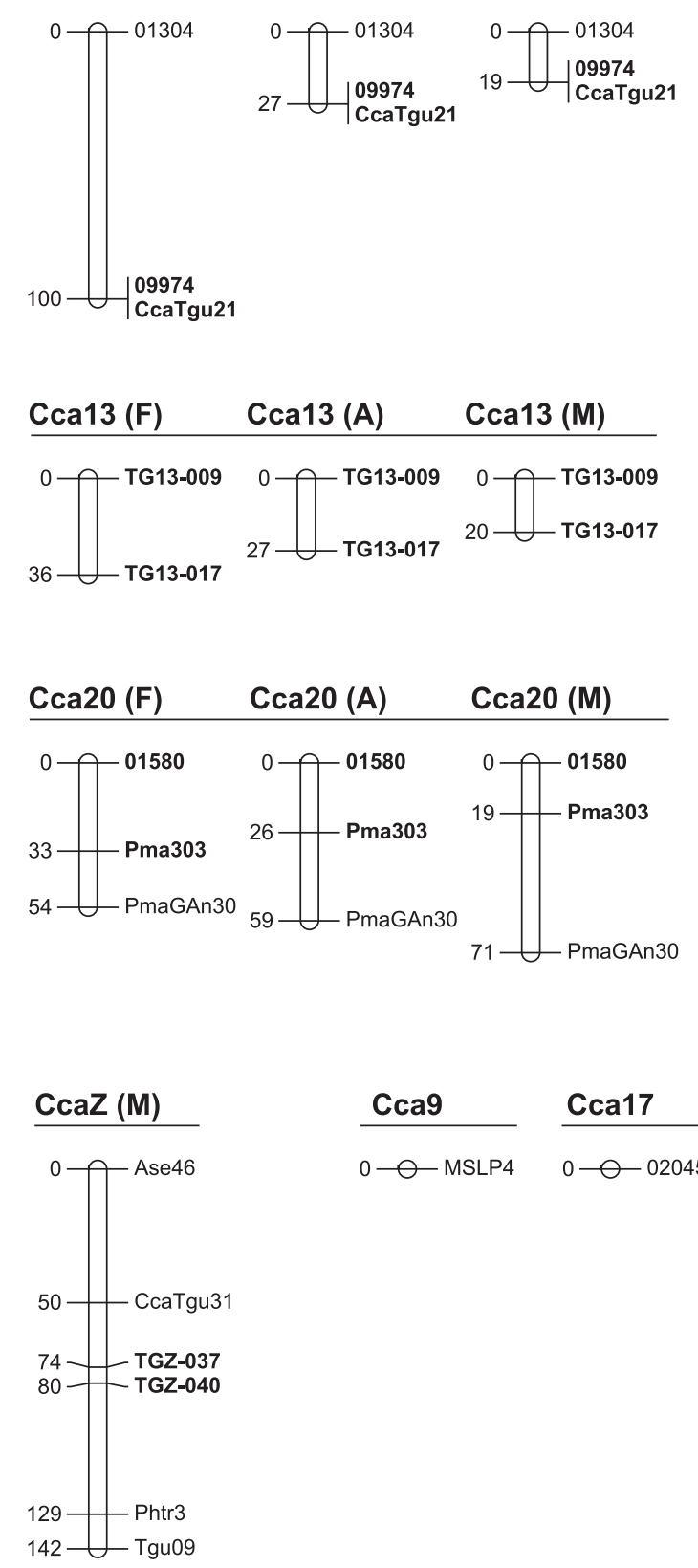

Figure 1 Continued.

linkage groups, $P=0.13$ ). The male-specific Z-linkage group was $142 \mathrm{cM}$.

The framework map (that is, the map including only the markers with unambiguous position) spanned $594 \mathrm{cM}$. The degree of heterochiasmy was lower than for the parsimonious map; the total framework map distance in females was $609 \mathrm{cM}$ and $567 \mathrm{cM}$ in males, that is, a female-to-male map ratio of 1.07 (Wilcoxon sign

\section{Cca10 (F) Cca10 (A) Cca10 (M)}

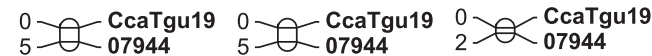

Cca12 (F) Cca12 (A) Cca12 (M)

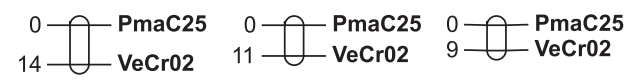

\section{$\operatorname{Cca15}(\mathrm{F}) \quad \operatorname{Cca15}(\mathrm{A}) \quad \operatorname{Cca15}(\mathrm{M})$

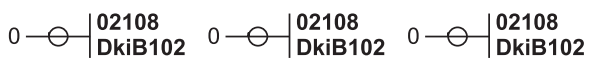

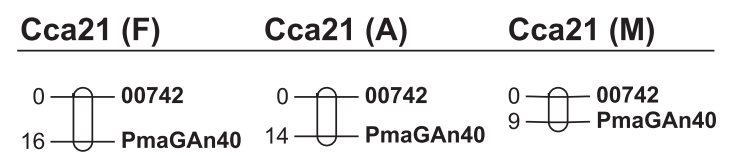

$\begin{array}{lll}\text { Cca18 } & \text { Cca22 } & \text { Cca28 } \\ 0-\ominus-D k i B 119 & 0-0-00227 & 0-0-04080\end{array}$

rank test: $z=0.84, n=18$ linkage groups, $P=0.40$ ). Only the two most central markers were framework loci on the Z-linkage group, with a distance of $5.5 \mathrm{cM}$ (Figure 2).

Synteny and gene order in blue tit, chicken and zebra finch

We found strong support for conserved synteny between chicken, zebra finch and blue tit. With few exceptions, 
markers in a specific blue tit linkage group had orthologous loci in a single chicken chromosome (Figure 2; Supplementary Table 1). Moreover, five of the nine markers that were unlinked in blue tit had orthologues located in unique chicken chromosomes (for example, MSLP4 was the only marker with an orthologue on Gga9; Figure 2; Supplementary Table 1). Accordingly, if there was conserved synteny, we would expect these five markers to segregate independently of other markers in the data set.
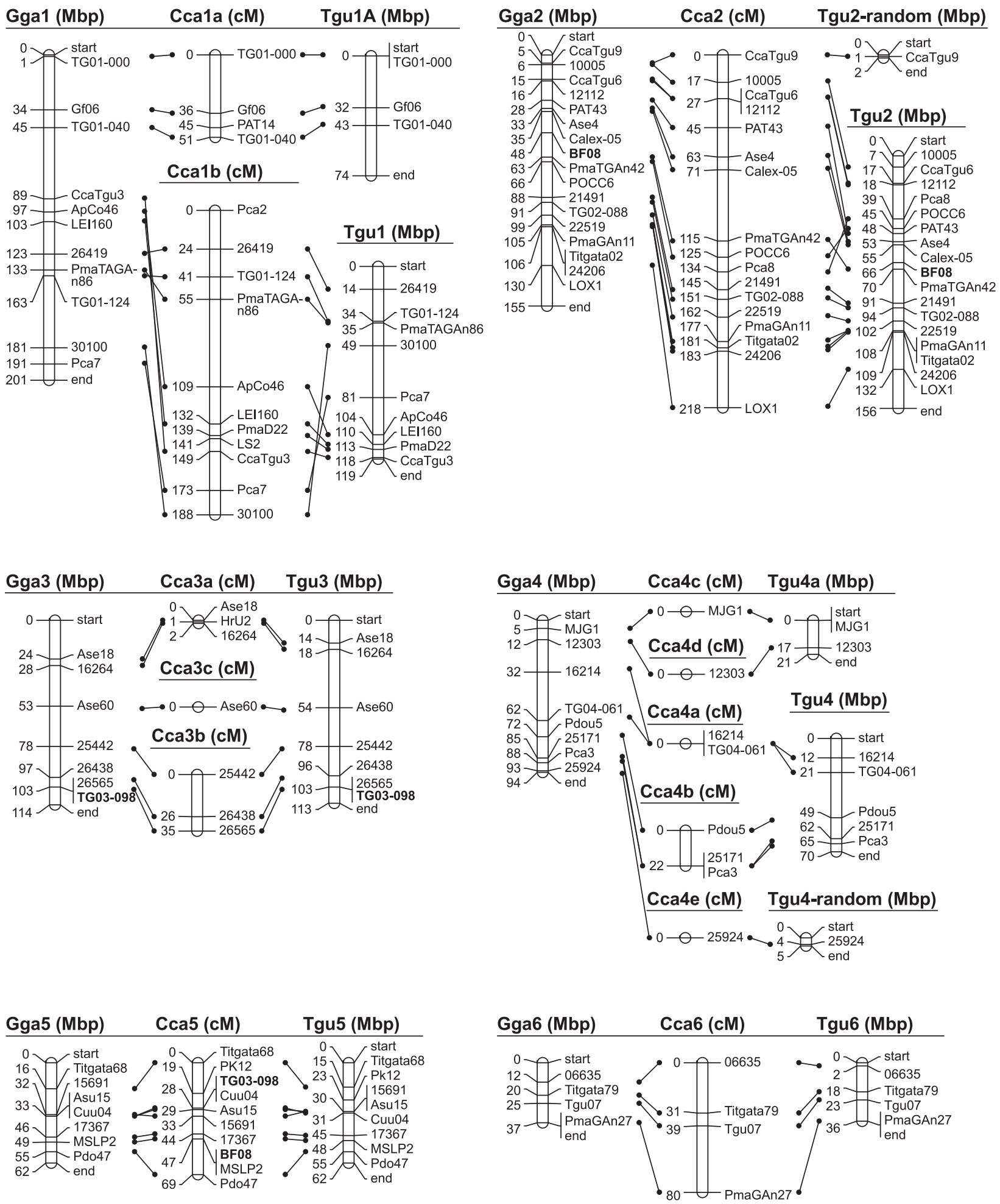

Figure 2 Linkage map distances for homologous chromosome regions in the blue tit (Cyanistes caeruleus; Cca in cM), and the position of orthologous loci in the chicken genome and zebra finch sequence assemblies ( $G g a$ and Tgu in Mbp). The position of all independently segregating loci in the blue tit is also given. The translocated markers, BF08 and TG03-098, are indicated (bold). 

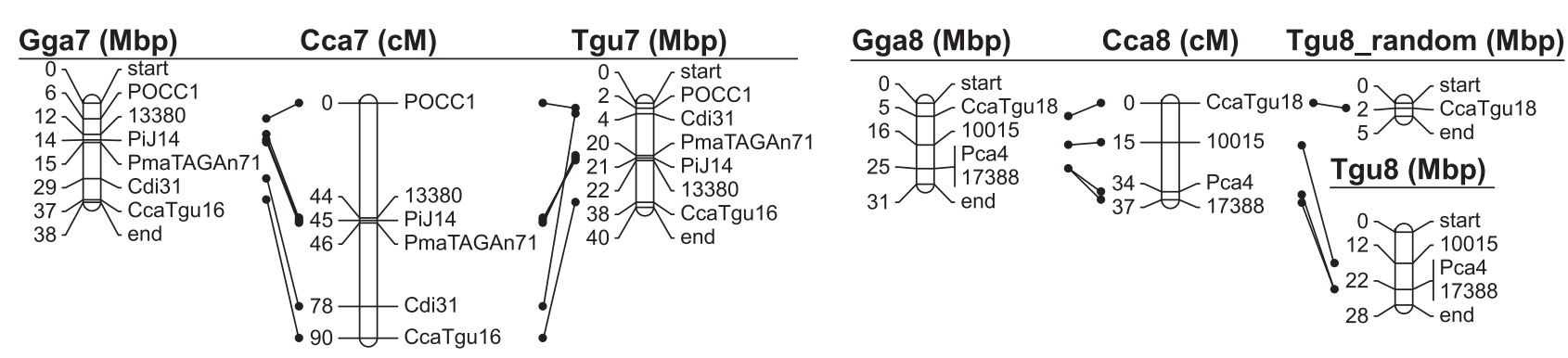

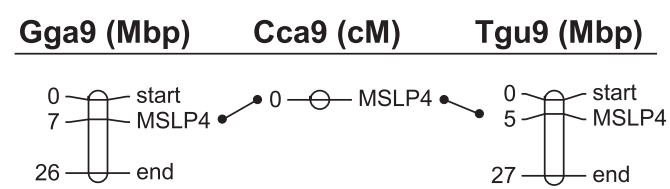

Gga11 (Mbp) Cca11 (cM) Tgu11 (Mbp)

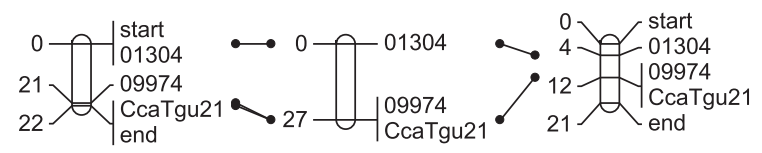

\section{Gga13 (Mbp) Cca13 (cM) Tgu13 (Mbp)}

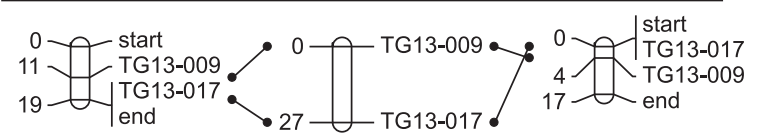

\section{Gga17 (Mbp) Cca17 (cM) Tgu17 (Mbp)}
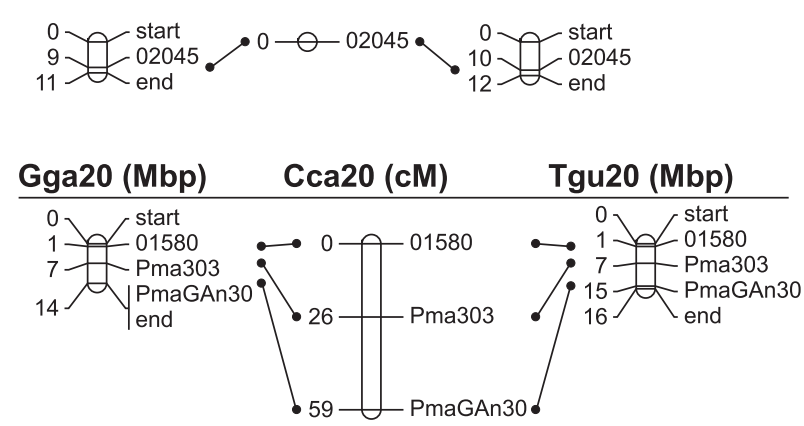

Gga22 (Mbp) Cca22 (cM) Tgu22 (Mbp)

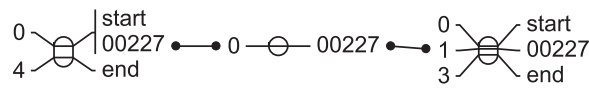

Gga28 (Mbp) Cca28 (cM) Tgu28 (Mbp)

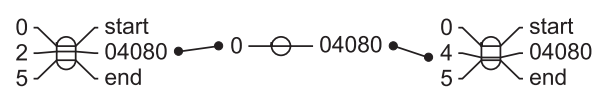

Figure 2 Continued.

The exceptions from conserved synteny were: (i) two blue tit chromosomes, $C c a 1 a$ and $C c a 1 b$, corresponded to a single chicken chromosome, Gga1; (ii) BF08 and TG03-
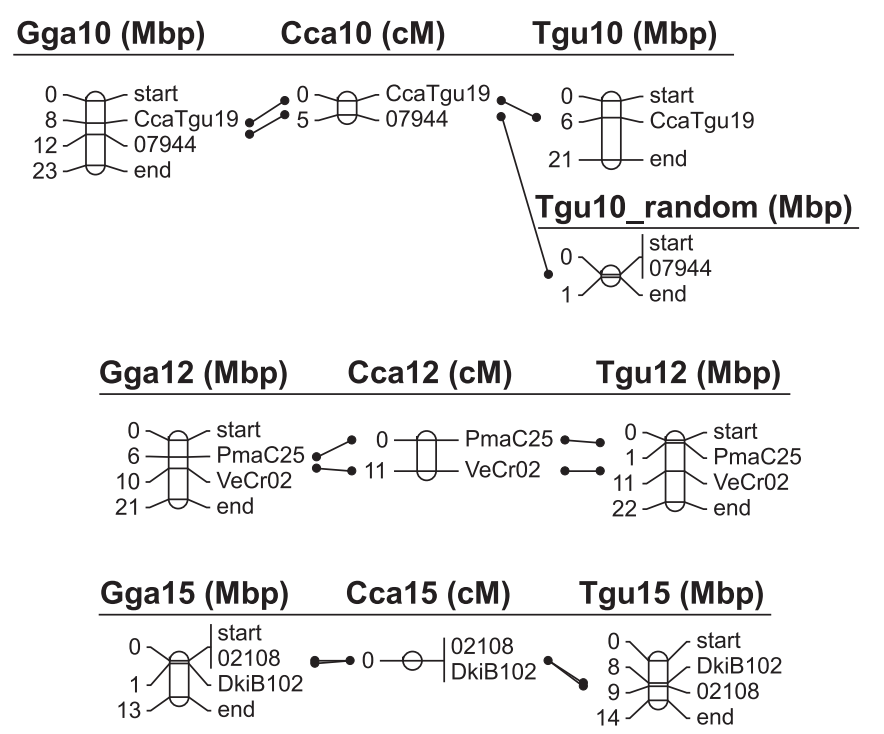

Gga18 (Mbp) Cca18 (cM) Tgu18_random (Mbp)
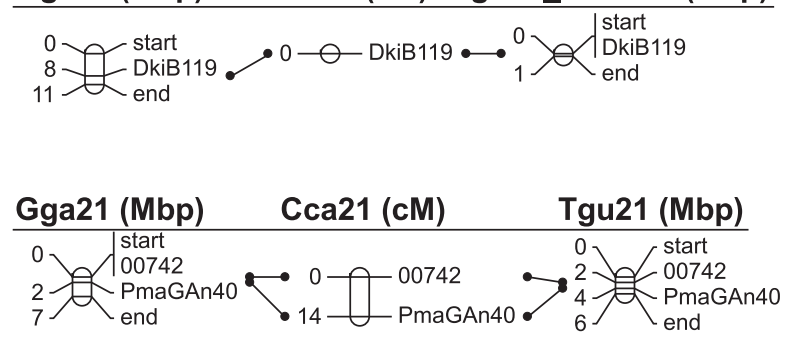

GgaZ (Mbp) $\quad$ CcaZ (cM) TguZ (Mbp)

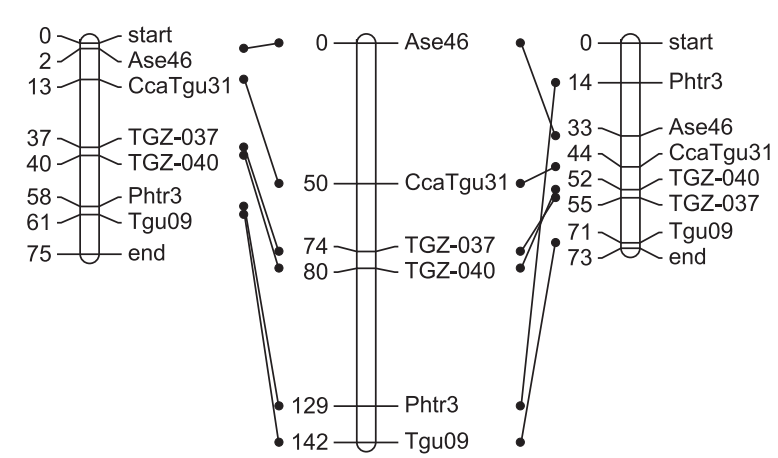

098 had orthologues on Gga2/Tgu2 and Gga3/Tgu3, respectively, but were both linked to markers on Cca5; (iii) Ase60 is found on Gga3 in chicken (Tgu3 in zebra 
finch) but was unlinked in the blue tit; and (iv) MJG1, 12303 and 25924 are found on Gga4 in chicken (Tgu4a and Tgu4_random in zebra finch) but were all unlinked in the blue tit. The allele sizes of the translocated loci, BF08 (range 101-117) and TG03-098 (range 238-264), in the blue tit matched that expected based on the sequenced clone (BF08: GenBank Accession Number AB091049, expected size $101 \mathrm{bp}$; TG03-098: DV573670, $235 \mathrm{bp}$ ), which supports that the targeted loci were amplified. Thus, although synteny is strongly conserved between chicken and blue tit, we documented a few cases of interchromosomal rearrangements.

The degree of gene-order conservation between blue tit and chicken varied from absolute correspondence of marker order (several chromosomes, for example, Cca2 vs Gga2) to several rearrangements ( $C c a 1 b$ vs Gga1; Figure 2). One unlinked marker (Ase60) and two linkage groups ( $\mathrm{Cca} 3 a$ and $\mathrm{Cca3b}$ ) in blue tit had a predicted location on chromosome 3 , and three unlinked markers (MJG1, 12303 and 25924) and two linkage groups (Cca4a and $C(a 4 b)$ in blue tit had a predicted location on chromosome 4 , but we could not confirm linkage between any of them in this study. This could have been due to low power to detect linkage because of some markers having low variability, low marker density and/ or high recombination rates, or the presence of chromosomal translocations.

\section{Recombination rate in blue tit and chicken}

This comparison included chromosomes 2, 5, 6, 7, 8, 11, 20 and $Z$, for which there were highly conserved synteny and gene order between chicken and blue tit (Figure 2), and substantial coverage ( $>50 \%$ of the chicken chromosome; Figure 2). In these homologous regions, the blue tit had shorter $(78 \%)$ parsimonious linkage map intervals than the Wageningen broiler chicken population (blue tit: $722 \mathrm{cM}$; chicken: $929 \mathrm{cM}$; Wilcoxon sign rank test: $z=1.54, n=8$ chromosomes, $P=0.12$ ). In contrast, there was no difference in map size between blue tit and the Uppsala chicken mapping population (709 cM; Wilcoxon sign rank test: $z=0.42, n=8$ chromosomes, $P=0.67)$.

\section{Discussion}

\section{Heterochiasmy}

Heterochiasmy is known from several animal and plant species. Haldane (1922) and Huxley (1928) hypothesized that in species in which sexes differ considerably in recombination rate, the heterogametic sex will have suppressed recombination. This is referred to as the 'Haldane-Huxley rule'. In support of this rule, reduced recombination rate in the heterogametic sex has been found in several mammals (for example, in human and mouse; Dietrich et al., 1996; Broman et al., 1998; Kong et al., 2002) and fish species (for example, in rainbow trout (Oncorhynchus mykiss); Sakamoto et al., 2000).

Stauss et al. (2003) studied recombination among three allozyme loci in a single linkage group in great tit (Parus major) and blue tit. Although genome-wide recombination rates should not be inferred from a single linkage group (Lynn et al., 2000; Sakamoto et al., 2000), their data indicated that heterochiasmy occurred in both these species, but in opposite directions; in the great tit, females had a higher recombination rate with a female- to-male recombination ratio of 1.91, whereas in the blue tit, males had more recombination with a female-to-male recombination ratio of $0.44-0.56$ (Stauss et al., 2003). In contrast, our results show that the blue tit also has higher recombination rate in females than in males, with a moderate degree of heterochiasmy (female-to-male map ratio of 1.07-1.18) at the genome-wide level. Some parts of the genome may have male-biased recombination, as suggested by the data in Stauss et al. (2003), and in some chromosomal regions in our linkage map, for example, between markers Pma303 and PmaGAn30 on Cca20.

In chicken the sexes have very similar map distances (Groenen et al., 2000, 2009), and this is also true for Japanese quail (Coturnix japonica; Kayang et al., 2004) and turkey (Meleagris gallopavo; Burt et al., 2003). The general pattern that is emerging from the passerine linkage mapping studies is that the degree of sex bias varies substantially between species, ranging from a pronounced female bias with a female-to-male map ratio of 1.5-2.1 in the great reed warbler (Hansson et al., 2005; Akkesson et al., 2007) to a moderate male bias in the collared flycatcher with a female-to-male map ratio of $\sim 0.8$ (Backström et al., 2008b). The other species studied to date, blue tit, zebra finch, Siberian jay (Perisoreus infaustus) and house sparrow (Passer domesticus, with data from a single chromosome), have an intermediate to low degree of heterochiasmy (this study; Hale et al., 2008; Stapley et al., 2008; Jaari et al., 2009). Females are the heterogametic sex in birds, and therefore the sex bias observed in some passerines-that females have higher recombination rate than males-opposes the HaldaneHuxley rule. Other firmly established exceptions to the Haldane-Huxley rule are found in marsupials, insects and plants (Lenormand, 2003; Samollow et al., 2004).

Consequently, there is no clear-cut association between heterogamety and heterochiasmy, and other (not necessarily exclusive) hypotheses have to be invoked to explain the phenomenon of sex-dimorphic map distances (Lenormand, 2003; Samollow et al., 2004; Hansson et al., 2005). These include (i) mechanistic explanations suggesting that heterochiasmy results from sex differences in metabolic rate or timing of meiosis (Bernstein et al., 1988; Plomion and O'Malley, 1996); (ii) hypotheses suggesting that sexual selection causes the sexes to diverge in recombination rate, because genes and combinations of genes that pass through the sex with highest variance in reproductive success would be, on average, superior, and thus selection would favour a reduced rate of recombination in this sex (Trivers 1988); and (iii) theories based on sex-specific epistatic gene interactions during the haploid phase or between chromosomes inherited from the father and the mother during the diploid phase (Lenormand 2003). Clearly, data on sex-specific recombination rates from more species, as well as data on sex-specific differences in metabolic rate, the strength of sexual selection, haploid gene expression and genomic imprinting, are needed to understand the observed variation in the degree of heterochiasmy in birds (Hansson et al., 2005).

\section{Synteny and gene order}

We found strong support for conserved synteny between blue tit and chicken. With a few exceptions, markers located on a specific blue tit linkage group had 
orthologues on a single chicken chromosome; and with the exception of one marker on Gga3 and three markers on Gga4, all unlinked markers had a predicted location on unique chromosomes. This result confirms the findings of previous studies of passerines: conserved synteny of several autosomes and the $\mathrm{Z}$ chromosome in the great reed warbler (Dawson et al., 2007), the zebra finch (Itoh et al., 2006; Stapley et al., 2008) and the collared flycatcher (Backström et al., 2006, 2008b), and conserved synteny at chromosome 7 in house sparrow (Hale et al., 2008). Thus, data from several species now support the conclusion of highly conserved synteny, with few interchromosomal rearrangements between Galliformes and Passeriformes.

Cytogenetic studies have shown that chicken chromosome 1 (Gga1) corresponds to two chromosomes in passerines (for example, Derjusheva et al., 2004; Griffin et al., 2007), and our data confirm this. In the blue tit, two independently segregating chromosomes, Cca1a and Cca1b, are homologous to different ends of Gga1. Comparative data suggest a fission of Gga1 in the lineage leading to passerines, and the split between Ccala and Cca1b in our data set occurs between markers TG01-040 and CcaTgu3, that is, between positions 45.2 and 88.7 Mbp of Gga1. This corroborates the findings in other species; in the great reed warbler, the split lies within 75.0 and $84.8 \mathrm{Mbp}$ of Gga1 (Dawson et al., 2007; B Hansson, unpublished data), in the zebra finch within 75.7 and $78.8 \mathrm{Mbp}$ of Gga1 (Stapley et al., 2008), and in the collared flycatcher within 68.6 and $80.3 \mathrm{Mbp}$ of $\mathrm{Gga1}$ (Backström et al., 2008b). Interestingly, Gga1 corresponds to three linkage groups in zebra finch and collared flycatcher, thus it is possible that additional fissions have occurred in passerines (Stapley et al., 2008; Backström et al., 2008b). In addition to the fission of Gga1 and Cca1a/ Cca1b, we detected that two loci (BF08 and TG03-098) had been translocated from their locations at Gga2/Tgu2 and Gga3/Tgu3, respectively, to chromosome 5. These two translocations are likely to include relatively small chromosomal segments, judging from the locations of markers adjacent to the predicted position of the translocated loci, predicting a maximum size of $27.5 \mathrm{Mbp}$ on Gga2 (region between 35.1 and $62.6 \mathrm{Mbp}$ ) and $10.6 \mathrm{Mbp}$ on Gga3 (region between 103.0 and 113.7 Mbp). These findings indicate that minor translocations have occurred in birds despite the overall wellconserved synteny in Passeriformes and Galliformes.

In general, the gene order within chromosomes seems to be highly conserved between chicken and passerines, although to a lesser extent than for synteny. The gene order of the most parsimonious blue tit map perfectly matched the gene order of chicken at several chromosomes (Cca1a, 2, 3b, 6, 7, 8, 20 and Z), and the support for conserved gene order was perhaps strongest for Gga2/ Cca2, with data for 16 orthologous markers and substantial coverage. Conserved gene order of Gga2 is, however, not a general pattern among passerines. For example, in the great reed warbler, there is a large inversion that covers at least $48.8 \mathrm{Mbp}$ of the central parts of that chromosome (region 58.6-107.4 Mbp; Dawson et al., 2007), and in the zebra finch and collared flycatcher, there are a few documented minor inversions (Stapley et al., 2008; Backström et al., 2008b).

We found support for at least two intrachromosomal rearrangements between Ggal and Cca1b. Similarly, both the great reed warbler and the zebra finch have an inversion in this part of Gga1, whereas this is not the case for the collared flycatcher (which may instead have a split on this part of Ggal as mentioned above; Backström et al., 2008b). Thus, there are at least two splits on chromosome 1 and several inversions, which indicate that this chromosome has been especially prone to accumulate rearrangements. Perhaps this suggests that intra- and interchromosomal rearrangement events do not occur independently.

Another interesting chromosome from a genome evolutionary point of view is Gga4. Cytogenetic studies suggest that Gga4 has been formed by a fusion of two ancestral avian chromosomes, and these two chromosomes are found in both Columbiformes (domestic pigeon Columba livia) and Passeriformes (chaffinch Fringilla coelebs; redwing Turdus iliacus; Derjusheva et al., 2004; Griffin et al., 2007). In line with this, Gga4 is represented by two linkage groups in the zebra finch: $T g u 4 a$ represents the first part of Gga4 (0-19 Mbp), and Tgu4 the end region (20-94 Mbp) (Stapley et al., 2008); moreover, a similar pattern is found in the collared flycatcher (Backström et al., 2008b). Two of the markers we mapped in the blue tit (MJG1 and 12303) lie within the region $0-19 \mathrm{Mbp}$ of Gga4 and on Tgu4a, and a fusion in the lineage leading to Galliformes could thus explain why these markers do not segregate with the other markers located on Gga4. However, it does not explain why MJG1 and 12303 are not tightly linked to each other. The other five markers that have orthologues in the region between 20 and $94 \mathrm{Mbp}$ of Gga4 do not form a single linkage group in our data set; instead, they form two linkage groups with two loci in each (Cca4a and $\mathrm{Cca} 4 \mathrm{~b}$ ). This may suggest that (i) some of these markers are located at different ends of the same chromosomes and have a high rate of recombination with the other markers (for example, 25924 is located at the end of Gga4); (ii) these markers are less informative (low statistical power in the linkage analyses), which seems unlikely as the number of informative meioses is similar to other markers; or (iii) there have been additional rearrangements of Gga4 over evolutionary time. As the marker density in our map is moderate, it is difficult to separate between these alternative explanations. By adding more markers to the map, we hope to address these issues in more detail in future research. Increasing the density of the linkage maps of the blue tit and other passerines is crucial to evaluate how frequently minor inversions are occurring in the Paridae and other passerine lineages.

The reason why the genome structure has been conserved to such a degree in birds (for example, Burt et al. (1999); Derjusheva et al. (2004); Dawson et al. (2007)), in contrast to what has been found in some mammalian lineages (Ferguson-Smith and Trifonov, 2007), and with the exception of the atypical and varying karyotypes among the birds of prey (Falconiformes; Bed'Hom et al., 2003; de Oliveira et al., 2005), has not been addressed in detail. Potential explanations include the possibility that the general paucity of repeat elements in the avian genome results in chromosome stability (International Chicken Genome Sequencing Consortium, 2004; Gordon et al., 2007), and that high dispersal rates and relatively large effective population sizes of birds slow down genome evolution (Hurst et al., 2004). Organisms with a 
large effective population size may be able to resist the spread of weakly deleterious mutations, and therefore are expected to have a more optimally organized genome (Lynch and Conery 2003).

An interesting aspect of genomic stability is the potential link between chromosomal rearrangements and the rate of speciation (Rieseberg 2001; Navarro and Barton, 2003; Price, 2008). Studies on, for example, Mus musculus domesticus have shown that crosses between chromosomal variants can result in hybrid inviability or impaired fitness (Capanna and Castiglia, 2004). The formation of post-zygotic incompatibility is substantially slower in birds than in mammals (Price and Bouvier, 2002; Fitzpatrick, 2004; Price, 2008), which may be explained by the slow rate of chromosomal rearrangement in birds.

\section{Inter-specific recombination rates}

The blue tit linkage map intervals are shorter than the Wageningen chicken broiler population (Groenen et al., 2009), similar to the Uppsala chicken mapping population (Wahlberg et al., 2007; Groenen et al., 2009), and larger than the great reed warbler (cf. Dawson et al., 2007). This could result from true inter-specific differences in recombination rate, but other explanations are also possible. First, it could be a consequence of the low density of the passerine maps, and that the linkage groups of some species are located in low-recombining parts of the genome, such as the centromeres (Hulten, 1974; Lynn et al., 2000). The recombination rate differs in different parts of the chicken genome and, as in mammals, recombination hot spots occur (Wahlberg et al., 2007; Groenen et al., 2009). However, this and other passerine linkage mapping studies, and the chicken linkage map, have had good coverage over compared chromosomes and the estimated level of recombination should not reflect rates in local chromosomal segments. Second, differences in linkage map intervals may reflect differences in genome sizes. However, although we cannot rule out this possibility completely, it is difficult to imagine drastic genome reductions in passerines without the loss of several functionally important genes, especially considering that chicken already has a comparatively compact genome (1.25 pg; Gregory, 2005). Moreover, the genome sizes of 45 other passerine species studied so far (mean $1.38 \mathrm{pg}$; range $1.04-1.93 \mathrm{pg}$ ) are comparable with, or at least not much smaller, than that of chicken (Gregory, 2005). Thus, it is likely that passerine species exhibit different recombination rates, and that some species have a lower recombination rate than domestic chicken. In fact, it is not surprising to find differences in recombination rates between phylogenetically diverged lineages, as it has been shown that the recombination rates can differ substantially even between closely related species and subspecies (True et al., 1996; Sanchez-Moran et al., 2002; Winckler et al., 2005). Recombination may evolve to optimize the allelic associations between loci as an epistatic response to natural selection; this process can shape the sex-average rate of recombination (reviewed in Otto and Lenormand (2002); Rice (2002)).

The recombination data from chicken (Groenen et al., 2009), blue tit (this study), great reed warbler (Dawson et al., 2007), collared flycatcher (Backström et al., 2008b) and zebra finch (Stapley et al., 2008) place domesticated chicken in one extreme with high rate of recombination and great reed warbler in the other extreme with remarkably low recombination rate. The Wageningen chicken broiler population has undergone strong directional selection, which may have selected for increased recombination as this may remove unfavourable genetic correlations and thus increase the selection response (Groenen et al., 2009). This cannot however explain the variation in recombination rate within passerines. Some models predict a higher recombination rate in inbred populations, and it has been observed cytologically that inbred plant species can have greater chiasma frequency compared with outcrossed relatives (Charlesworth et al., 1977, 1979; Hansson et al., 2006). However, the particular low recombination rate in great reed warblers cannot be explained by a hypothesis based on inbreeding, as the studied population in central Sweden is fairly new and small, and has been going through a recent bottleneck (Bensch et al., 2000; Hansson et al., 2000); in addition, phylogeographical studies indicate Pleistocene bottlenecks in the species (Bensch and Hasselquist, 1999; Hansson et al., 2008). Thus, if anything, the great reed warbler would be expected to be more inbred than, for example, the blue tit, and therefore would be expected to have higher recombination rate, which is clearly not the case. Alternative hypotheses include that the processes causing the pronounced heterochiasmy in great reed warblers (Hansson et al., 2005; Åkesson et al., 2007) have simultaneously caused a general decline in the recombination rate in this species compared with other passerines.

\section{Conclusions}

The emerging view from this and previous avian mapping studies (Dawson et al., 2007; Stapley et al., 2008; Backström et al., 2008b) is that the physical map of the chicken (International Chicken Genome Sequencing Consortium, 2004; http://www.ncbi.nlm.nih.gov/ mapview /) and the recently released zebra finch genome assembly (http://genome.wustl.edu/genomes/view/ taeniopygia_guttata/; http://www.ncbi.nlm.nih.gov/ mapview/) provide very useful resources for predicting the genome organization and chromosome content in passerines, with a caveat of potential difficulties to predict and generalize the degree of gene-order conservation for different chromosomes within and between species (cf. Dawson et al., 2006, 2007; Stapley et al., 2008; Backström et al., 2008b). Passerine linkage mapping is still in its infancy, with low-density linkage maps, and large regions of the genome are still to be comparatively analysed in passerines. Therefore, it seems likely that future studies using high-density linkage maps will identify additional rearrangements, especially minor inversions and translocations, both between Passeriformes and Galliformes, and between different species of Passeriformes.

\section{Acknowledgements}

We thank Johan Nilsson and Martin Stjernman for their efforts in the field and Gavin Horsburgh for technical assistance. Terry Burke kindly provided comments on the paper. The work was supported by the Swedish Research Council (to BH and JAN), Nils-Olof Berggren's foundation 
(to $\mathrm{BH}$ and ML), Lunds Djurskyddsfond (to ML) and the Natural Environment Research Council, UK (to DAD).

\section{References}

Åkesson M, Hansson B, Hasselquist D, Bensch S (2007). Linkage mapping of AFLP markers in a wild population of great reed warblers: importance of heterozygosity and number of genotyped individuals. Mol Ecol 16: 2189-2202.

Backström N, Brandstrom M, Gustafsson L, Qvarnström A, Cheng $\mathrm{H}$, Ellegren $\mathrm{H}$ et al. (2006). Genetic mapping in a natural population of collared flycatchers (Ficedula albicollis): conserved synteny but gene order rearrangements on the avian Z chromosome. Genetics 174: 377-386.

Backström N, Fagerberg S, Ellegren H (2008a). Genomics of natural bird populations: a gene-based set of reference markers evenly spread across the avian genome. Mol Ecol 17: 964-980.

Backström N, Karaiskou N, Leder EH, Gustafsson L, Primmer CR, Qvarnström A et al. (2008b). A gene-based genetic linkage map of the collared flycatcher (Ficedula albicollis) reveals extensive synteny and gene-order conservation during 100 million years of avian evolution. Genetics 179: 1479-1495.

Bed'Hom B, Coullin P, Guillier-Gencik Z, Moulin S, Bernheim A, Volobouev V et al. (2003). Characterization of the atypical karyotype of the black-winged kite Elanus caeruleus (Falconiformes: Accipitridae) by means of classical and molecular cytogenetic techniques. Chromosome Res 11: 335-343.

Bensch S, Hasselquist D (1999). Phylogeographic population structure of great reed warblers: an analysis of mtDNA control region sequences. Biol J Linn Soc 66: 171-185.

Bensch S, Hansson B, Hasselquist D, Nielsen B (2000). Partial albinism in a semi-isolated population of great reed warblers. Hereditas 133: 167-170.

Bernstein H, Hopf F, Michod R (1988). Is meiotic recombination and adaptation for repairing DNA, producing genetic variability, or both?. In: $\mathrm{R}$ Michod, B Levin (eds). The Evolution of Sex. Sinauer: Sunderland, MA. pp 139-160.

Blondel J, Dias PC, Perret P, Maistre M, Lambrechts MM (1999). Selection-based biodiversity at a small spatial scale in a lowdispersing insular bird. Science 285: 1399-1402.

Broman KW, Murray JC, Sheffield VC, White RL, Weber JL (1998). Comprehensive human genetic maps: Individual and sex-specific variation in recombination. Am J Hum Genet 63: 861-869.

Burt DW, Bruley C, Dunn IC, Jones CT, Ramage A, Law AS et al. (1999). The dynamics of chromosome evolution in birds and mammals. Nature 402: 411-413.

Burt DW, Morrice DR, Sewalem A, Smith J, Paton IR, Smith EJ et al. (2003). Preliminary linkage map of the Turkey (Meleagris gallopavo) based on microsatellite markers. Anim Genet 34: 399-409.

Capanna E, Castiglia R (2004). Chromosomes and speciation in Mus musculus domesticus. Cytogenet Genome Res 105: 375-384.

Charlesworth D, Charlesworth B, Strobeck C (1979). Selection for recombination in self-fertilising species. Genetics 93: 237-244.

Charlesworth D, Charlesworth B, Strobeck C (1977). Effects of selfing on selection for recombination. Genetics 68: 213-226.

Charmantier A, Kruuk LE, Blondel J, Lambrechts MM (2004). Testing for microevolution in body size in three blue tit populations. J Evol Biol 17: 732-743.

Cramp S (1992). Handbook of the birds of Europe, the Middle East and North Africa Vol VI Oxford University Press: Oxford.

Dawson DA, Akesson M, Burke T, Pemberton JM, Slate J, Hansson B (2007). Gene order and recombination rate in homologous chromosome regions of the chicken and a passerine bird. Mol Biol Evol 24: 1537-1552.

Dawson DA, Burke T, Hansson B, Pandhal J, Hale MC, Hinten GN et al. (2006). A predicted microsatellite map of the passerine genome based on chicken-passerine sequence homology. Mol Ecol 15: 1299-1320.

de Oliveira EH, Habermann FA, Lacerda O, Sbalqueiro IJ, Wienberg J, Müller S (2005). Chromosome reshuffling in birds of prey: the karyotype of the world's largest eagle (Harpy eagle, Harpia harpyja) compared to that of the chicken (Gallus gallus). Chromosoma 114: 338-343.

Derjusheva S, Kurganova A, Habermann F, Gaginskaya E (2004). High chromosome conservation detected by comparative chromosome painting in chicken, pigeon and passerine birds. Chromosome Res 12: 715-723.

Dietrich WF, Miller J, Steen R, Merchant MA, Damron-Boles D, Husain $\mathrm{Z}$ et al. (1996). A comprehensive genetic map of the mouse genome. Nature 380: 149-152.

Ellegren $\mathrm{H}$ (2005). The avian genome uncovered. Trends Ecol Evol 20: 180-186.

Ellegren H, Sheldon BC (2008). Genetic basis of fitness differences in natural populations. Nature 452: 169-175.

Ferguson-Smith MA, Trifonov V (2007). Mammalian karyotype evolution. Nat Rev Genet 8: 950-962.

Fitzpatrick BM (2004). Rates of evolution of hybrid inviability in birds and mammals. Evolution 58: 1865-1870.

Foerster K, Delhey K, Johnsen A, Lifjeld JT, Kempenaers B (2003). Females increase offspring heterozygosity and fitness through extra-pair matings. Nature 425: 714-717.

Gordon L, Yang S, Tran-Gyamfi M, Baggott D, Christensen M, Hamilton A et al. (2007). Comparative analysis of chicken chromosome 28 provides new clues to the evolutionary fragility of gene-rich vertebrate regions. Genome Res 17: 1603-1613.

Gratten J, Wilson AJ, McRae AF, Beraldi D, Visscher PM, Pemberton JM et al. (2008). A localized negative genetic correlation constrains microevolution of coat colour in wild sheep. Science 319: 318-320.

Gregory TR (2005). Animal Genome Size Database. http:// www.genomesize.com

Griffin DK, Robertson LB, Tempest HG, Skinner BM (2007). The evolution of the avian genome as revealed by comparative molecular cytogenetics. Cytogenet Genome Res 117: 64-77.

Groenen MA, Crooijmans RP, Veenendaal A, Cheng HH, Siwek M, van der Poel JJ (1998). A comprehensive microsatellite linkage map of the chicken genome. Genomics 49: 265-274.

Groenen MA, Wahlberg P, Foglio M, Cheng HH, Megens HJ, Crooijmans RP et al. (2009). A high-density SNP-based linkage map of the chicken genome reveals sequence features correlated with recombination rate. Genome Res 19 510-519.

Groenen MA, Cheng HH, Bumstead N, Benkel BF, Briles WE, Burke T et al. (2000). A consensus linkage map of the chicken genome. Genome Res 10: 137-147.

Haldane JBS (1922). Sex ratio and unisexual sterility in hybrid animals. J Genet 12: 101-109.

Hale MC, Jensen H, Birkhead TR, Burke T, Slate J (2008). A comparison of synteny and gene order on the homologue of chicken chromosome 7 between two passerine species and between passerines and chicken. Cytogenet Genome Res 121 120-129.

Hansson B, Åkesson M, Slate J, Pemberton JM (2005). Linkage mapping reveals sex-dimorphic map distances in a passerine bird. Proc R Soc Lond B 272: 2289-2298.

Hansson B, Bensch S, Hasselquist D, Lillandt BG, Wennerberg $\mathrm{L}$, von Schantz $\mathrm{T}$ (2000). Increase of genetic variation over time in a recently founded population of great reed warblers (Acrocephalus arundinaceus) revealed by microsatellites and DNA fingerprinting. Mol Ecol 9: 1529-1538.

Hansson B, Hasselquist D, Tarka M, Zehtindjiev P, Bensch S (2008). Postglacial colonisation patterns and the role of isolation and expansion in driving diversification in a passerine bird. PLoS One 3: e2794.

Hansson B, Kawabe A, Preuss S, Kuittinen H, Charlesworth D (2006). Comparative gene mapping in Arabidopsis lyrata chromosomes 1 and 2 and the corresponding $A$. thaliana 
chromosome 1: recombination rates, rearrangements and centromere location. Genet Res 87: 75-85.

Hulten M (1974). Chiasma distribution at diakinesis in normal human male. Hereditas 76: 55-78.

Hurst LD, Pal C, Lercher MJ (2004). The evolutionary dynamics of eukaryotic gene order. Nat Rev Genet 5: 299-310.

Huxley JS (1928). Sexual differences of linkage in Gammarus chevreuxi. I Genet 20: 145-156.

International Chicken Genome Sequencing Consortium (2004). Sequencing and comparative analysis of the chicken genome provide unique perspectives on vertebrate evolution. Nature 432: 695-716.

Itoh Y, Arnold AP (2005). Chromosomal polymorphism and comparative painting analysis in the zebra finch. Chromosome Res 13: 47-56.

Itoh Y, Kampf K, Arnold AP (2006). Comparison of the chicken and zebra finch $\mathrm{Z}$ chromosomes shows evolutionary rearrangements. Chromosome Res 14: 805-815. e-pub ahead of print January 192007.

Jaari S, Li MH, Merila J (2009). A first-generation microsatellitebased genetic linkage map of the Siberian jay (Perisoreus infaustus): insights into avian genome evolution. BMC Genomics 10: 1 .

Kayang BB, Vignal A, Inoue-Murayama M, Miwa M, Monvoisin JL, Ito $S$ et al. (2004). A first-generation microsatellite linkage map of the Japanese quail. Anim Genet 35: 195-200.

Kong A, Gudbjartsson DF, Sainz J, Jonsdottir GM, Gudjonsson SA, Richardsson B et al. (2002). A high-resolution recombination map of the human genome. Nat Genet 31: 241-247.

Kvist L, Broggi J, Illera JC, Koivula K (2005). Colonisation and diversification of the blue tits (Parus caeruleus teneriffaegroup) in the Canary Islands. Mol Phylogenet Evol 34: 501-511.

Lander ES, Green P (1987). Construction of multilocus genetic-linkage maps in humans. Proc Natl Acad Sci USA 84: 2363-2367.

Lenormand $\mathrm{T}$ (2003). The evolution of sex dimorphism in recombination. Genetics 163: 811-822.

Lenormand T, Dutheil J (2005). Recombination difference between sexes: a role for haploid selection. PLoS Biol 3: e63.

Lynch M, Conery JS (2003). The origins of genome complexity. Science 302: 1401-1404.

Lynn A, Kashuk C, Petersen MB, Bailey JA, Cox DR, Antonarakis SE et al. (2000). Patterns of meiotic recombination on the long arm of human chromosome 21. Genome Res 10: 1319-1332.

Lyons LA, Laughlin TF, Copeland NG, Jenkins NA, Womack JE, O'Brien SJ (1997). Comparative anchor tagged sequences (CATS) for integrative mapping of mammalian genomes. Nat Genet 15: 47-56.

Masabanda JS, Burt DW, O'Brien PCM, Vignal A, Fillon V, Walsh PS et al. (2004). Molecular cytogenetic definition of the chicken genome: the first complete avian karyotype. Genetics 166: 1367-1373.

Nager RG, Wiersma P (1996). Physiological adjustment to heat in blue tit Parus caeruleus nestlings from a Mediterranean habitat. Ardea 84: 115-125.

Navarro A, Barton NH (2003). Chromosomal speciation and molecular divergence-accelerated evolution in rearranged chromosomes. Science 300: 321-324.

Nilsson J-Å, Svensson E (1993). Energy constraints and ultimate decisions during egg-laying in the blue tit. Ecology 74: 244-251.

Olano-Marin J, Dawson DA, Girg A, Hansson B, Ljungqvist M, Kempenaers B et al. (2009). A genome-wide set of 106 microsatellite markers for the blue tit (Cyanistes caeruleus). Mol Ecol Resour, submitted.

Otto SP, Lenormand T (2002). Resolving the paradox of sex and recombination. Nat Rev Genet 3: 252-261.
Petrie M, Kempenaers B (1998). Extra-pair paternity in birds: explaining variation between species and populations. Trends Ecol Evol 13: 52-58.

Plomion C, O'Malley DM (1996). Recombination rate differences for pollen parents and seed parents in Pinus pinaster. Heredity 77: 341-350.

Price T (2008). Speciation in Birds. Roberts and Company: Greenwood Village, Colorado.

Price TD, Bouvier MM (2002). The evolution of F1 postzygotic incompatibilities in birds. Evolution 56: 2083-2089.

Råberg L, Stjernman M, Hasselquist D (2003). Immune responsiveness in adult blue tits: heritability and effects of nutritional status during ontogeny. Oecologia 136: 360-364.

Rice WR (2002). Experimental tests of the adaptive significance of sexual recombination. Nat Rev Genet 3: 241-251.

Rieseberg LH (2001). Chromosomal rearrangements and speciation. Trends Ecol Evol 16: 351-358.

Sakamoto T, Danzmann RG, Gharbi K, Howard P, Ozaki A, Khoo SK et al. (2000). A microsatellite linkage map of rainbow trout (Oncorhynchus mykiss) characterized by large sex-specific differences in recombination rates. Genetics 155: 1331-1345.

Samollow PB, Kammerer CM, Mahaney SM, Schneider JL, Westenberger SJ, VandeBerg JL et al. (2004). First-generation linkage map of the gray, short-tailed opossum, Monodelphis domestica, reveals genome-wide reduction in female recombination rates. Genetics 166: 307-329.

Sanchez-Moran E, Armstrong SJ, Santos JL, Franklin FC, Jones GH (2002). Variation in chiasma frequency among eight accessions of Arabidopsis thaliana. Genetics 162: 1415-1422.

Shetty S, Griffin DK, Graves JA (1999). Comparative painting reveals strong chromosome homology over 80 million years of bird evolution. Chromosome Res 7: 289-295.

Shields GF (1982). Comparative avian cytogenetics. Condor 84: $45-58$.

Stapley J, Birkhead TR, Burke T, Slate J (2008). A linkage map of the zebra finch Taeniopygia guttata provides new insights into avian genome evolution. Genetics 179: 651-667.

Stauss M, Tomiuk J, Segelbacher G, Driesel S, Fietz J, Bachmann $L$ et al. (2003). Sex-specific recombination rates in Parus major and $P$ caeruleus, an exception to Huxley's rule. Hereditas 139: 199-205.

Steiner CC, Weber JN, Hoekstra HE (2007). Adaptive variation in beach mice produced by two interacting pigmentation genes. PLOS Biol 5: e219.

Stjernman M, Råberg L, Nilsson J-Å (2004). Survival costs of reproduction in the blue tit (Parus caeruleus): a role for blood parasites? Proc $R$ Soc Lond B 271: 2387-2394.

Sturtevant AH (1913). The linear arrangement of six sex-linked factors in Drosophila, as shown by their mode of association. I Exp Zool 14: 43-59.

Trivers $\mathrm{R}$ 1988. Sex differences in rates of recombination and sexual selection. In: R Michod, B Levin (eds). The Evolution of Sex. Sinauer: Sunderland, MA. pp 270-286.

True JR, Mercer JM, Laurie CC (1996). Differences in crossover frequency and distribution among three sibling species of Drosophila. Genetics 142: 507-523.

van Tuinen M, Sibley CG, Hedges SB (2000). The early history of modern birds inferred from DNA sequences of nuclear and mitochondrial ribosomal genes. Mol Biol Evol 17: 451-457.

Wahlberg P, Stromstedt L, Tordoir X, Foglio M, Heath S, Lechner D et al. (2007). A high-resolution linkage map for the $\mathrm{Z}$ chromosome in chicken reveals hot spots for recombination. Cytogenet Genome Res 117: 22-29.

Winckler W, Myers SR, Richter DJ, Onofrio RC, McDonald GJ, Bontrop RE et al. (2005). Comparison of fine-scale recombination rates in humans and chimpanzees. Science 308: 107-111.

Supplementary Information accompanies the paper on Heredity website (http://www.nature.com/hdy) 